
\title{
R Research Soure \\ Molecular and FISH analysis of 45S rDNA on BAC molecule of Saccharina Japonica
}

\author{
Peng-Fei Liu \\ Shanghai Ocean University \\ Yan-Hui Bi \\ Shanghai Ocean University \\ Li Liu \\ Shanghai Ocean University \\ Zhi-Gang Zhou ( $\nabla$ zgzhoushou@163.com ) \\ Shanghai Ocean University
}

\section{Research Article}

Keywords: Saccharina japonica, BAC library, 45s rDNA, IGS, Fiber-FISH

Posted Date: June 1st, 2021

DOI: https://doi.org/10.21203/rs.3.rs-527149/v1

License: (9) This work is licensed under a Creative Commons Attribution 4.0 International License. Read Full License

Version of Record: A version of this preprint was published at Aquaculture and Fisheries on September 1st, 2021. See the published version at https://doi.org/10.1016/j.aaf.2021.07.002. 


\section{Abstract}

IGS is abundant in polymorphism, which is widely used in the analysis of intraspecific genetic diversity and phylogenetic relationships among geographical populations. In this study, the 45S rDNA repeat unit of Saccharina japonica was obtained for the first time by BAC clone sequencing. The total length of $45 S$ rDNA repeat unit of $S$. japonica was 8995 bp, including 5420 bp of 18s-5.8s-25s rDNA and 3575 bp of IGS (Intergenic Spacer), with the GC content of $51.4 \%$. IGS was composed of 465 bp 3'-outer transcribed spacer (ETS), 874 bp 5'-ETS, and 2236 bp non transcribed spacer (NTS), with the GC content of $50.1 \%$.Fiber-FISH (fiber-fluorescence in situ hybridization, fiber-FISH) analysis of $45 \mathrm{~S}$ rDNA on the BAC molecule of female gametophytes of $S$. japonica illustrated that each fiber had at least five continuous moniliform hybridization signal points, indicating the distribution of 45S rDNA repeat unit on the bacterial artificial chromosome. This study provided a new candidate molecular marker for detecting intraspecific polymorphisms of S. japonica, and the successful fiber-FISH analysis of 45S rDNA on BAC molecule would contribute to the construction of the physical map and Map-based cloning of this kelp.

\section{Introduction}

In eukaryotes, ribosomal 45S rRNA genes (rDNA) are located in the nucleolar organizer region (NOR) of chromosomes in the form of tandem repeats [1-3]. Each 45S rDNA repeat unit is composed of ribosomal gene coding regions (25S, 5.8S and 18S rDNA), two internal transcribed spacer (ITS) and one intergenic spacer (IGS) [4]. Due to different natural selection pressures, 45s rRNA demonstrated different genetic development among different species and even high intraspecific genetic diversity. IGS is located between the 3-terminal of 25s rRNA gene and the 5-terminal of 18S rRNA gene. IGS contains a 3' external transcribed spacer (ETS), a 5'-ETS, a non transcribed spacer (NTS), on which there is a transcription initiation site (TIS), a transcription termination site (TTS) and a series of regulatory sequences. In comparision with the conservation of rRNA gene, IGS is highly polymorphic and has been widely used in species classification and identification $[3,5]$.

Due to the complicated structure of IGS, including the high GC content, self-complementary structure and the repeated sequence, it is difficult to obtain the full-length sequence of IGS by PCR [6]. Till now, only few IGS full-length sequenceshad been reported from macroalgae including Pyropia yezoensis[7, 8], Pyropia haitanensis[9, 10], Bangia [11], Ulva prolifera and Blidingia sp [12]. Bacterial artificial chromosome (BAC) is an engineered DNA molecule in bacterial cells. Large segments ranging from $100 \mathrm{~kb}$ to about $300 \mathrm{~kb}$ of an organism could be inserted into BACs for whole genome sequencing [13], physical map construction $[14]$, and map based cloning $[15,16]$ due to its high genetic stability.

Saccharina japonica is an economically and ecologically important brown alga. So far, no 45S rDNA complete repeat unit had been reported. On the basis of BAC library of female gametophyte of $S$. japonica, the BAC clone containing 45S rDNA repeat unit was screened and sequenced by using the third generation of PacBio sequencing technology. In addition, with the 18S rDNA sequence of $S$. japonica as a probe, the 45S rDNA repeat unit was successfully located in bacterial artificial chromosome by using 
fiber-FISH. This was the first report about fluorescent in situ hybridization of molecular marker on DNA fibers in macroalgae. The results would contribute to the construction of physical map and map-based cloning of $S$. japonica and other macroalgae.

\section{Materials And Methods}

\subsection{Materials}

The female gametophyte BAC library of $S$. japonica was constructed and preserved in our laboratory. It contains $31872 \mathrm{BAC}$ monoclones with an average length of $115 \mathrm{~kb}$, covering 6.57 times of the whole genome of $S$. japonica gametophyte.

\subsection{Methods}

\subsubsection{BAC Clone screening and sequencing}

For screening BAC clones containing $45 \mathrm{~S}$ rDNA repeat units, three-dimensional PCR was conducted with primers designed with the 18S rRNA gene of S. japonica (GenBank accession number:EU293553) as template. The primers were 18F: 5'- tcggacggtttgtggtga-3' and 18R: 5'- ccttccttggatggtggtagcc-3'. 25 $\mathrm{L}$ PCR reaction system included $12.5 \mu \mathrm{L} 2 \times$ dream Taq PCR mix [0.1 U/ $\mu \mathrm{L}$ Taq polymerase, $500 \mu \mathrm{mol} / \mu \mathrm{L}$ dNTP, 20 mmol/L Tris-HCl (pH 8.3), $100 \mathrm{mmol} / \mathrm{L} \mathrm{KCl,} 3$ mmol/L MgCl 2 ] (Thermo Fisher Scientific Inc., USA), $0.5 \mu \mathrm{L}$ upstream and $0.5 \mu \mathrm{L}$ downstream primers of $10 \mu \mathrm{mol} / \mathrm{L}, 1 \mu \mathrm{L}$ bacterial solution as template DNA. The amplification procedure included $94^{\circ} \mathrm{C}$ pre denaturation for $3 \mathrm{~min}$, followed by 30 cycles of $94^{\circ} \mathrm{C}$ denaturation for $45 \mathrm{~s}, 57^{\circ} \mathrm{C}$ annealing for $45 \mathrm{~s}, 72^{\circ} \mathrm{C}$ extension for $1.5 \mathrm{~min}$, and $72^{\circ} \mathrm{C}$ extension for 10 min. The products of PCR were detected by $1 \%$ agarose gel electrophoresis.

The high molecular weight BAC plasmid DNA of the screened clones was extracted witha DNA Extraction Kit (Qiagen, Germany).Pulsed field gel electrophoresis was performed for measure the size of the inserted DNA fragment after Notl digestion of the extracted plasmid. The inserted fragments were sequenced using the third generation of PacBio sequencing technology (Wuhan Eight Star Bio-tech Co., Ltd., Wuhan, China).

\subsubsection{DNA Sequence analysis of BAC clone}

The obtained sequence was pre-processed to remove cloning vector sequences from the reads using Vec Screen(https://www.ncbi.nlm.nih.gov/tools/vecscreen/) of NCBI. Sequence similarity analysis was conducted by using Blastnhhttps://blast.ncbi.nlm.nih.gov/Blast.cgin. The Plant-CARE database (http://bioinformatics.psb.ugent.be/webtools/plantcare/html/) was employed to predict the conserved cis-element motifs presented in IGS. CpG islands in the IGS were predicted by using CpGPlot [17]. The physical map of the inserted fragment of the positive BAC clone (625-E18) was constructed according to the genome sequenceof $S$. japonicaby using IBS1.0 [18] and MapChart2.32 [19].

1.2.3 FISH on BAC clone molecule 
The positive BAC clones were cultured in LB liquid medium containing $12.5 \mu \mathrm{g} / \mathrm{mL}$ chloramphenicol. The BAC plasmid DNA was extracted with plasmid DNA Extraction Kit (Qiagen, Germany). $5 \mu \mathrm{L}$ BAC-DNA (about $50 \mathrm{ng}$ ) was diluted in $5 \mu \mathrm{L}$ sterilized deionized water and then pipette onto a poly-L-lysine glass slide. A $18 \mathrm{~mm} \cdot 18 \mathrm{~mm}$ coverslip was used to spread the molecular to prevent molecular broken [20]. Slide was air-dried for $30 \mathrm{~min}$ at room temperature, and then the coverslip was washed off in water. After air dry, slide was fixed in the Carnot's fixative (Ethanol / glacial acetic, 3:1, v/v) [21] for $2 \mathrm{~min}$, and dried at $60^{\circ} \mathrm{C}$ for $30 \mathrm{~min}$.

The 18S rDNA probes were labeled by nick-translation with Alex flow green-5-dUTP (PerkinElmer, Boston, USA). FISH was performed according to the procedure described by Liu et al. [22].Slide with BAC-DNA was cross-linked for 2-3 times in a ultraviolet cross linker, and then $8 \mu \mathrm{L}$ of hybridization solution, including $6 \mu \mathrm{L}$ of mixture ( $2 \times \mathrm{SSC}+1 \times \mathrm{TE}$ buffer) and $2 \mu \mathrm{L}$ of $18 \mathrm{~S}$ rDNA probe, was pipette on the slide. After covering with a coverslip, the slide was water bath at $100^{\circ} \mathrm{C}$ for $5 \mathrm{~min}$. Then put it in an oven at $55^{\circ} \mathrm{C}$ for hybridization overnight. After hybridization, the slide was washed with $2 \times \mathrm{SSC}$ at $42{ }^{\circ} \mathrm{C}$ for 10 min and then dried at $55^{\circ} \mathrm{C}$ for $15 \mathrm{~min}$. At last, $5 \mu \mathrm{L}$ DAPI staining solution was added. After incubation for $10 \mathrm{~min}$, the results were observed under a fluorescence microscope. LeicaDM4000 fluorescence microscope (Germany) and Orca ER camera were used to take pictures, and Adobe PhotoShop CS6.0 was used to process the pictures.

\section{Results And Analysis}

\subsection{Characterization of the BAC clone harbouring 45S rDNA}

With 18S rRNA gene as probe, one BAC clone (625-E18) containing 18S rRNA gene was successfully screened from the BAC library of female gametophyte of $S$. japonica by three-dimensional PCR (Fig. S1). The result of PFGE showed that the inserted fragment of this positive clone was about $95 \mathrm{~kb}$ in length (Fig. 1).

In total, 181233 reads were derived from the third generation of PacBio sequencing results, with an average length of $3990 \mathrm{bp}$ (Fig. 2). After splicing, an inserted DNA fragment with $24437 \mathrm{bp}$ was obtained. On the basis of Blastn alignment results, three DNA fragments between 6074 bp-7895 bp, 15069 bp$16890 \mathrm{bp}$ and $21510 \mathrm{bp}-23331 \mathrm{bp}$ on the sequenced region of BAC clone 625-E18 were matched with $18 \mathrm{~S}$ rRNA genes of $S$. japonica gametophytes. These results implied that the sequenced region of BAC clone 625-E18 harbouredthree 45S rDNA repeat units, and each repeat unit was 8995 bp in length, which included 5420 bp of 18 S-5.8S-25S rDNA sequence and 3575 bp of IGS.

\subsection{Sequence characterization of IGS ofS. japonica}

The size of IGS of S. japonica was 3575 bp in length, which was constituted by 465 bp of $3^{\prime}$-outer transcribed spacer (ETS), 874 bp of 5'-ETS and 2236 bp of non transcribed spacer (NTS) in turn (Fig. 3). The GC content of the IGS sequence was $50.1 \%$. A transcription termination site (TTS) was predicted in 
the NTS, which was rich in cytosine (CCCCCCCCCCCCCA), and was highly consistent with the reported 25 s rRNA TTS of higher plants $[23,24]$. It was speculated to participate in the termination of $S$. japonica rRNA gene transcription. At 2702-2713 bp of 5'-ETS, a transcription initiation site (TIS,TTTTTAGGGGGG) was predicted, of which the base A at 2707 bp might be the RNA transcription initiation site. In addition, three $\mathrm{CpG}$ islands were predicted in the IGS.

On the basis of the basic local alignment search results, apart from the two fragments of $6756 \mathrm{bp}-6827$ bp and 9366 bp-9435 bp in Scaffold4565, the sequenced region of BAC clone (625-E18) matched well with the sequence between $785 \mathrm{bp}$ and $10030 \mathrm{bp}$ of Scaffold4565 of S. japonica genome (Fig. 3), which accounted for $75 \%$ of the total length of Scaffold4565 [25]. In the physical map of the sequenced region of BAC clone 625-E18, it was noted thatthe IGS and 25S rDNA in the third 45S rDNA unit were slightly shorter than the other two. In addition, the region between 9435 bp-10030 bp in Scaffold 4565 could not be matched with the corresponding 25S rRNA gene sequence of $S$. japonica (Fig. 3). These results illustrated that BAC clone sequencing and the physical map construction were efficient way of the genome sequence error diagnosis and thus improving the accuracy of genome sequences.

\subsection{FISH on BAC molecule}

With $18 \mathrm{~S}$ rDNA as probe, 45S rDNA repeat units were successfully visualized on the BAC 625-E18 molecular fiber by FISH. FISH results illustrated that the hybridization signal points representing repeat units were lined like continuous moniliform on the molecular fiber (Fig. 4A). On the selected three molecular fibers, five, seven and seven signal points were visualized respectively as shown in Fig. 4B, Fig. 4C and Fig. 4D. These results implied that BAC clone 625-E18 harboured at least seven 45s rDNA repeat units.

\section{Discussions}

\subsection{IGS sequence of $S$. japonica}

In this study, the full-length IGS sequence of $S$. japonica was $3575 \mathrm{bp}$, which was the first report of IGS in brown algae. IGSs were more variable than protein coding genes such as $\mathrm{nr}$ SSU, 5.8S rDNA, and $\mathrm{nr}$ LSU in 45S rDNA[26]. The length of IGS varied greatly among different organisms, which was from about $1 \mathrm{~kb}$ in yeast to $12 \mathrm{~kb}$ in plant [27]. In macroalgae, only few IGS full length sequences had been documented. Generally, the reported sizes of IGSs of red algae were longer than that of $S$. japonica, and they were 5984 bp, 6969 bp and 4613 bp in Pyropia yezoensis [8], P. haitanensis [9], and Bangia [11], respectively. In contrast, the IGSs of green algae were slightly shorter than that of S. japonica, such as the IGSs of Ulva proliferaand Blidingia sp, which were $3388 \mathrm{bp}$ and $3059 \mathrm{bp}$ in length [12], respectively. According to that IGS was more suitable for phylogenetic analysis at the level of genus, species and subspecies [24], the full length sequence of IGS reported in this paper would provide useful molecular marker for phylogenetic analysis at the level of subspecies of this kelp. 
In contrast to the high variability of the sequence length of IGS, its structure and function were relatively conserved. All the IGS contained a TTS, a TIS and some transcriptional regulatory elements [5]. In the IGS of $S$. japonica, the sequence of TTS was composed of CCCCCCCCCCCCCA, of which the characteristic of pyrimidine enrichment was also common in the higher plants $[23,24]$. The TIS of $S$. japonica was composed of TTTTTAGGGGGG. It had been reported that the sequences from -5 to +6 at both ends of TIS sequence had important functions and were highly invariant [28]. In most plants, TATA-box and GGGG-box were common elements at the 5 'end of rRNA precursor. There was a GGGG-box downstream of the transcription initiation site of $S$. japonica TIS, but the TATA-box was instead of TTTTT upstream. This phenomenon of TATA-box variation was also described in the higher plants, such as Punica granatum $L$ and Quercus palustris Münchh.,in which TATA-box was instead of TCTTT [24, 29]. In addition, the IGS of $S$. japonica had some methylation sites (three $\mathrm{CpG}$ islands). In plants, cytosine in CpGロCpNpG and $\mathrm{CpHp}$ was prone to methylation, which leaded to gene silencing. The expression of rRNA gene was regulated by ribosome requirement. In actively growing cells, the transcription of rRNA accounted for the majority of the total RNA, while in the cells with inactive growth, the transcription of rRNA gene was greatly reduced, indicating that cells mainly regulated the synthesis of rRNA on the level of transcription. DNA methylation and post transcriptional modification of histidine were considered to be involved in gene silencing. There were plenty of repeats in ribosomal genes, and the methylation of CpG in IGS region was considered to be enough to inhibit the transcription of rRNA gene [30]. Three CpG islands in the IGS sequence of $S$. japonica were identified in this study, which also might participate in the regulating the transcription of rRNA gene through DNA methylation.

\subsection{Distribution of 45S rDNAsitesin BACmolecule}

In this study, at least seven $45 S$ rDNA repeat units of $S$. japonica were localized in BAC molecule. This is the first report about the cytological localization of DNA molecular of macroalgae by fiber-FISH. Ribosomes play an important role in protein synthesis and cell growth. rRNA accounts for $50 \%-80 \%$ of total RNA of cells [31,32]. Correspondingly, there are tandem repeats of rDNA in the nucleolar organizer region of one or more chromosomes [33].The nuclear genome of eukaryotes may contain hundreds to thousands of rRNA gene repeats. For macroalgae, there was no report on the number of 45S rDNA repeat units yet. In this study, three $45 \mathrm{~S}$ rDNA repeat units were screened on the basis of BAC contig sequencing, and at least seven 45S rDNA sites were localized in BAC molecule by fiber-FISH. Although the number of $45 S$ rDNA repeat units deduced from this study might be lower than actual number, the molecular and cytological analysis of $45 \mathrm{~S}$ rDNA of $S$. japonica in this study would provide new candidate molecular markers for the study of the genetic diversity of S. japonica, and the successful conduction of fiber-FISH on BAC molecule would contribute to the construction of the physical map and Map-based cloning of $S$. japonica and other macroalgae.

\section{Declarations}

Funding 
This work was supported by the National Natural Science Foundation of China (grant No. 41376136 to Z.G. Z.), the National Key Research and Development Program of China (grant No. 2018 YFD0901500 to Y.H. B.) and World Class Discipline Project of Aquaculture (to Z.-G. Z.).

Availability of data and material

The datasets used or analysed during the current study are available from the corresponding author on reasonable request.

Code availability (software application or custom code)

Not applicable.

Author Contributions

P.-F. Liu has carried out most experiments. Y.-H. Bi analyzed all these data and confirmed the results in addition to the writing of this manuscript. Li Liu has conducted three-dimensional PCR for screening of the BAC library. Z.-G. Zhou has made a significant contribution to the conceptual design of the work.

Ethical approval: This article does not contain any studies with human participants performed by any of the authors.

\section{References}

1. Roa F, Guerra M (2012) Distribution of 45S rDNA sites in chromosomes of plants: structural and evolutionary implications. BMC Evol Biol 12:225. 10.1186/1471-2148-12-225

2. Grabiele M, Debat HJ, Moscone EA et al (2012) )25S-18S rDNA IGS of Capsicum: molecular structure and comparison. Plant Syst Evol 298(2):313-321. 10.1007/s00606-011-0546-8

3. Gruendler P, Unfried K, Pascher K, Schweizer D (1991) rDNA intergenic region from Arabidopsisthaliana: structural analysis, intraspecific variation and functional implications. J Molec Biol 221:1209-1222. 10.1016/0022-2836(91)90929-Z

4. Poczai P, Hyvönen J (2010) Nuclear ribosomal spacer regions in plant phylogenetics: problems and prospects. Mol Biol Rep 37(4):1897-1912. 10.1007/s11033-009-9630-3

5. Volkov RA, Bachmair A, Panchuk II, Kostyshyn SS, Schweizer D (1999) 25S-18S rDNA intergenic spacer of Nicotiana Sylvestris (Solanaceae): primary and secondary structure analysis. Plant Syst Evol 218:89-97. 10.1007/BF01087037

6. Yang K, Robin A, Yi GE, Lee J, Chung MY, Yang TJ, Nou IS (2015) Diversity and inheritance of intergenic spacer sequences of 45S ribosomal DNA among accessions of Brassica oleracea L. var. capitate.Int. J Mol Sci 16:28783-28799. 10.3390/ijms161226125

7. Li X, Xu J, He Y, Shen S, Zhu J, Shen Z (2016) The complete nuclear ribosomal DNA (nrDNA) cistron sequence of Pyropiayezoensis (Bangiales, Rhodophyta). J Appl Phycol 28(1):663-669. 
8. He Y, Xu J, Li X, Shen S, Zhu J, Shen Z, Jiang B, Lu Q (2015)Ribosomal intergenic spacer (IGS) sequence can distinguish varieties of Pyropiayezoensis cultivated in China. Botanica Marina 2015. 58(6): 465-473. 10.1515/bot-2015-0032

9. He Y, Shen S, Shen Z (2017) Cloning and application of the complete nuclear ribosomal DNA (nrDNA) cistron sequence of Pyropiahaitanensis(Bangiales, Rhodophyta). Bot Mar 60(3):327-337. 10.1515/bot-2016-0079

10. Li Y, Shen S, He L, Xu P, Lu S (2010) Sequence analysis of rDNA intergenic spacer (IGS) of Porphyrahaitanensis. J Appl Phycol 22(2):187-193. 10.1007/s10811-009-9441-x

11. Xu J, Jiang B, Chai S, He Y, Zhu J, Shen Z, Shen S (2016) Complete nuclear ribosomal DNA sequence amplification and molecular analyses of Bangia (Bangiales, Rhodophyta)from China. Chin JOceanol Limn 34(5):1044-1053. 10.1007/s00343-016-5033-1

12. Shen WJ, Yuan H, Shen SD (2019) A new molecular label applied to the study of the Yellow Sea green tide. J Ocean Univ China 18(6):1507-1514. 10.1007/s11802-019-3988-1

13. Derelle E, Ferraz C, Lagoda $P$ et al (2002)DNA libraries for sequencing the genome of Ostreococcustauri (Chlorophyta, Prasinophyceae): the smallest free-living eukaryotic cell.J Phycol38(6): 1150-1156. 10.1046/j.1529-8817.2002.02021.x

14. Maruyama S, Misumi O, Ishii Y et al (2004) The minimal eukaryotic ribosomal DNA units in the primitive red alga Cyanidioschyzonmerolae. DNA Res 11(2):83-91. 10.1093/dnares/11.2.83

15. Nguyen RL, Tam LW, Lefebvre PA (2005) The LF1 gene of Chlamydomonasreinhardtii encodes a novel protein required for flagellar length control. Genetics 169(3):1415-1424.

10.1534/genetics.104.027615

16. Hamaji T, Ferris PJ, Nishii I et al (2009) Identification of the minus mating-type specific gene MTD1 from Goniumpectorale (Volvocales, Chlorophyta). J Phycol 45(6): 1310-1314. 10.1111/j.15298817.2009.00744.x

17. Rice P, Longden I, Bleasby A (2000) EMBOSS: The European molecular biology open software suite. Trends Genet 16:276-277. 10.1016/s0168-9525(00)02024-2

18. Liu W, Xie Y, Ma J et al (2015) IBS: an illustrator for the presentation and visualization of biological sequences. Bioinformatics 31(20):3359-3361. 10.1093/bioinformatics/btv362

19. Voorrips RE (2002) )MapChart: Software for the graphical presentation of linkage maps and QTLs. J Hered 93(1):77-78. 10.1093/jhered/93.1.77

20. Wang K, Zhang W, Jiang Y et al (2013) Systematic application of DNA fiber-FISH technique in Cotton. PLoS One 8(9):e75674. 10.1371/journal.pone.0075674

21. Schweizer D (1976) Reverse fluorescent chromosome banding with chromomycin and DAPI. Chromosoma 58(4):307-324. 10.1007/BF00292840

22. Liu Y, Bi Y-H, Gu J-G et al (2012) Localization of a female-specific marker on the chromosomes of the brown seaweed Saccharina japonica using fluorescence in situ hybridization.PLoS One7(11): 
e48784. 10.1371/journal.pone.0048784

23. Kelly RJ, Siegel A (1989) The Cucurbita maxima ribosomal DNA intergenic spacer has a complex structure. Gene 80(2):239-248. 10.1016/0378-1119(89)90288-6

24. Parvaresh M, Talebi M (2014) Molecular characterization of ribosomal DNA intergenic spacer (IGS) region in pomegranate (Punica granatum L.). Plant Syst Evol 300(5):899-908. 10.1007/s00606-0130928-1

25. Ye N, Zhang X, Miao M et al (2015) Saccharina genomes provide novel insight into kelp biology. Nat Commun 6:6986. 10.1038/ncomms7986

26. Harper JT, Saunders GW (2001) The application of sequencesof the ribosomal cistron to the systematics and classification of the florideophyte red algae (Florideophyceae, Rhodophyta). Cah Biol Mar 42:25-38

27. Kim KJ, Mabry TJ (1991) Phylogenetic and evolutionary implicationsof nuclear ribosomal DNA variation in dwarf dandelions (Krigia,Lactuceae, Asteraceae). Plant Syst Evol 177:53-69. 10.1007/BF00937826

28. Doelling JH, Pikaard CS (1995) The minimal ribosomal RNA gene promoter of Arabidopsis thalianaincludes a critical element at the transcription initiation site. Plant J 8(5):683-692. 10.1046/j.1365-313X.1995.08050683.x

29. Bauer N, Horvat T, Biruš I,Vičić V,Zoldoš V (2009) Nucleotidesequence, structural organization and length heterogeneity ofribosomal DNA intergenic spacer in Quercus petraea (Matt.) Liebl. and Q. roburL. Mol Genet Genomics 281:207-221. 10.1007/s00438-008-0404-8

30. Reeder R (1989) Regulatory elements of the generic ribosomal gene. Curr Opin Cell Biol 1(3):466474. 10.1016/0955 - 0674(89)90007-0

31. Moss T, Stefanovsky VY (1995) Promotion and regulation of ribosomal transcription in eukaryotes by RNA polymerase I. Prog Nucleic Acid Res Mol Biol 50:25-66. 10.1016/S0079-6603(08)60810-7

32. Paule MR, Lofquist AK (1996) Organization and expression of eukaryotic ribosomal RNA genes. In: Zimmermann RA, Dahlberg AE (eds) Ribosomal RNA Structure, Evolution, Processing and Function in Protein Biosynthesis. CRC Press, Boca Raton, pp 395-419

33. Mateos M, Markow TA (2005) Ribosomal intergenic spacer (IGS) length variation across the Drosophilinae (Diptera: Drosophilidae). BMC Evol Biol 5:46. 10.1186/1471-2148-5-46

\section{Figures}




\section{$12 \quad \mathbf{M}$}

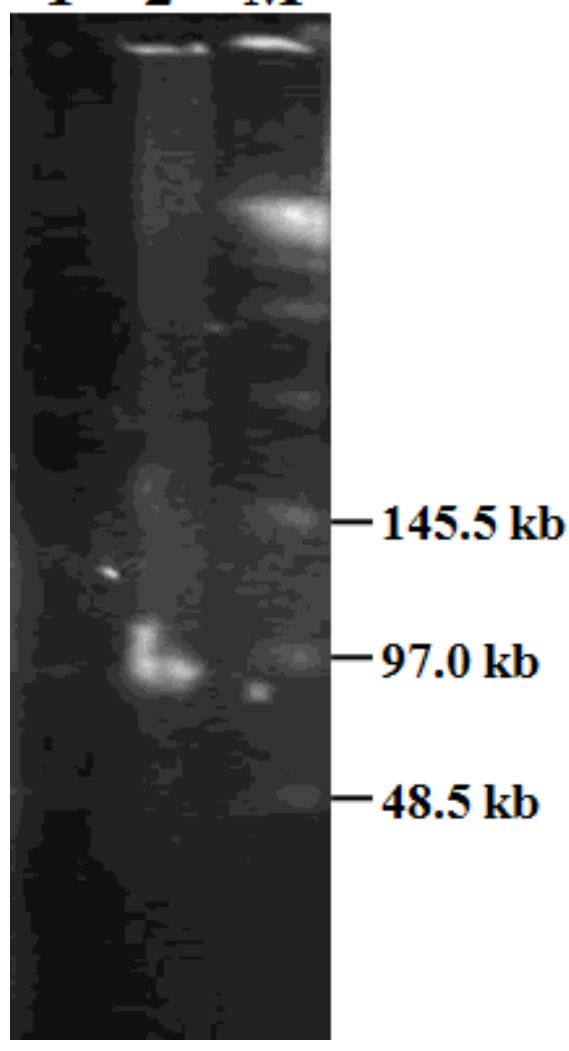

\section{Figure 1}

1Pulsed-field gel electrophoresis (PFGE) profile of BAC625-E18 from the BAC library of the S. japonica female gametophytes. Lane 1: control with $\mathrm{H} 20$ instead of BAC clone DNA; Lane 2: Notl-digested DNA of the screened BAC625-E18 from the BAC library of S. japonica female gametophytes; Lane M: $\lambda$ Ladder PFG marker (New England Biolabs). 


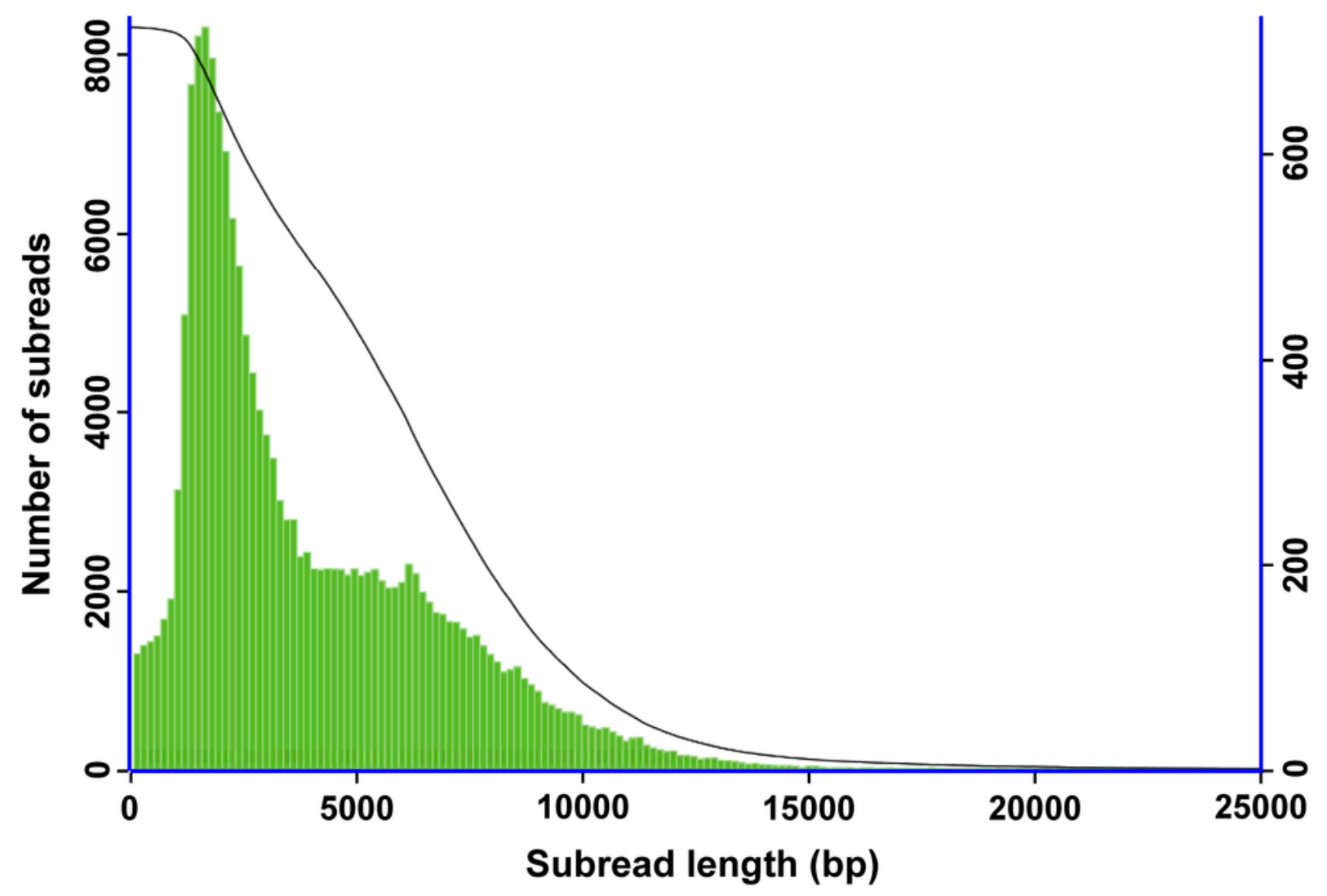

Figure 2

Distribution of read length and number of the sequenced BAC625-E18. 


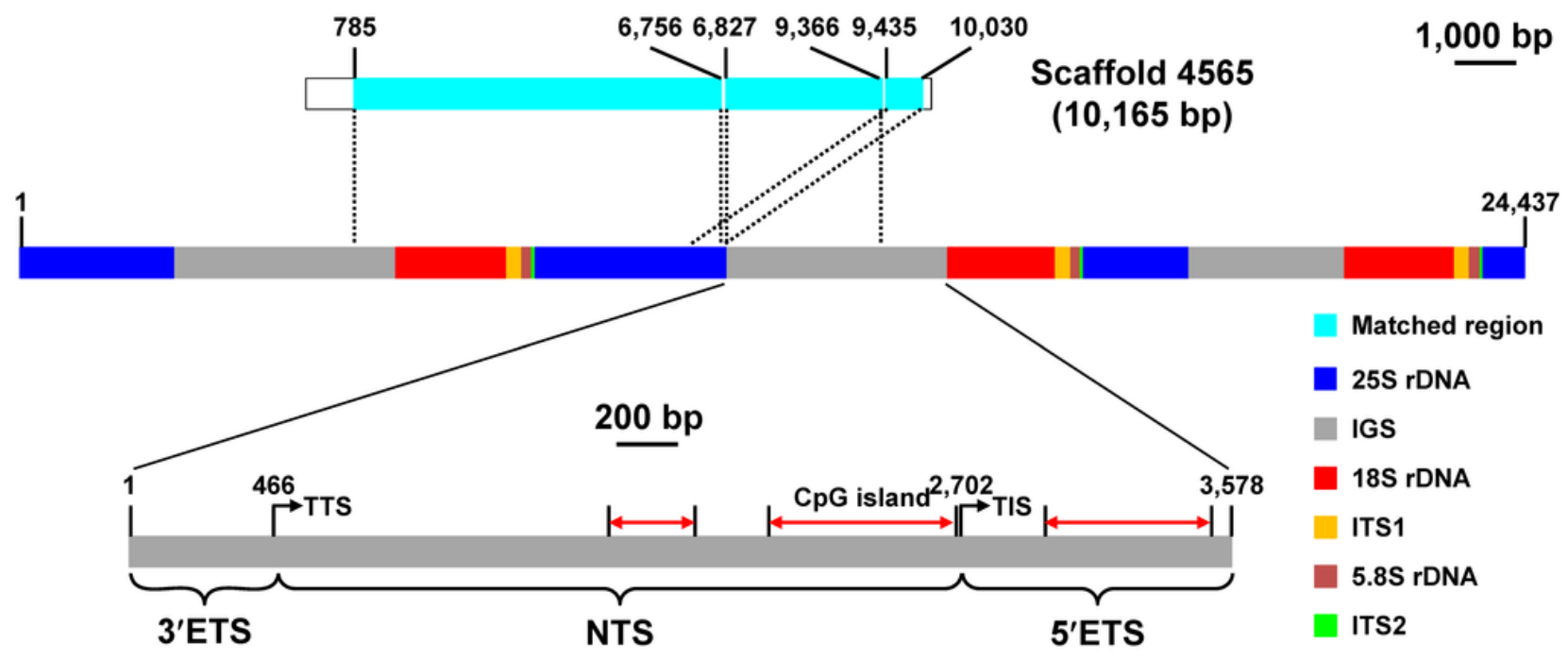

Figure 3

Physical map of BAC clone 625-E18 sequence (lower) and its location in the assembled scaffold of S. japonica (upper). 


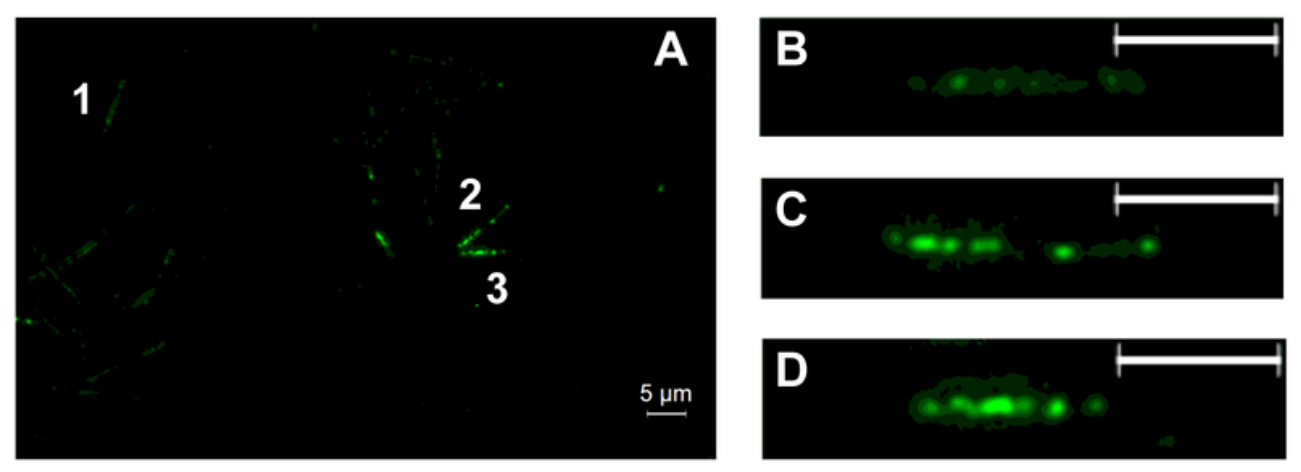

\section{Figure 4}

Fluorescence insitu hybridization on the extracted vectors from BAC clone 625-E18 with the labaled S.japonica $18 \mathrm{~S}$ rDNA as a probe (green). Images B, C and D are the enlarged signals which are marked 1, 2 and 3 , respectively, in Image A; Bar corresponds to $5 \mu \mathrm{m}$.

\section{Supplementary Files}

This is a list of supplementary files associated with this preprint. Click to download.

- OnlineFigureS1.png 